\title{
A FIXED POINT THEOREM
}

\author{
B. O'NEILL AND E. G. STRAUS ${ }^{1}$
}

1. The fixed point theorem. Let $T: X \rightarrow Y$ be a point-to-set function and let $T^{-1}: Y \rightarrow X$ be the point-to-set function such that $x \in T^{-1}(y)$ if and only if $y \in T(x)$. If $X$ and $Y$ are topological spaces, $T$ is continuous provided the function $T^{-1}$ is both open and closed, and each set $T(x)$ is closed.

Let $H$ be Cech homology theory with coefficients in a field, and let $E_{n}$ be Euclidean $n$-space. A mapping $f: B \rightarrow E_{n}$ will be said to link a point of $E_{n}$ provided that $x \notin f(B)$ and the induced homomorphism $f_{*}: H_{n-1}(B) \rightarrow H_{n-1}\left(E_{n}-x\right)$ is not zero.

THEOREM. Let $C$ be a compact connected subspace of $E_{n}$ and let $T: C \rightarrow C$ be a continuous point-to-set function. Let $B$ be a compact space, $I$ the unit interval, with $h: B \times I \rightarrow E_{n}$ a homotopy such that $h_{0}$ links every point of $C$ and $h_{1}$ links no point of $C$. Then there exists a number $t$ with $0<t \leqq 1$ and a point $y \in C$ such that $y \in h_{t}(B)$ and $h_{t}(B)$ meets $T(y)$.

(Here, as usual, $h_{t}$ denotes the map of $B$ into $E_{n}$ such that $h_{t}(b)$ $=h(b, t))$. We note that the hypothesis on $h$ is fulfilled in case $B$ is the boundary of a bounded open set containing $C$ and $h$ is a deformation of $B$ such that $E_{n}-h_{1}(B)$ is connected.

To prove the theorem we need the following lemmas.

Lemma 1 (Notation as in Theorem). In the space $C \times E_{1}$, let $U=\{(x, t) \mid t<0\}, V=\{(x, t) \mid t>1\}, B^{*}=\{(x, t) \mid x=h(b, t)$ for some $b \in B\}$. Then $U$ and $V$ are contained in different components of $C \times E_{1}-B^{*}$.

Proof. Let $L$ be the set of points $(x, t)$ of $C \times I$ such that $h_{t}$ links $x$. In view of the hypotheses on $h_{0}$ and $h_{1}$, it suffices to prove that $L$ and $C \times I-\left(B^{*} \cup L\right)$ are open in $C \times I$. If $(x, t) \in C \times I-B^{*}$, there is an $\epsilon$-neighborhood $N$ of $x$ in $E_{n}$ and a neighborhood $P$ of $t$ in $I$ such that if $u \in P$ then $h_{u}(B) \cap N=\phi$. Consider the neighborhood $(N \cap C) \times P$ of $(x, t)$ in $C \times I$. If $(y, u)$ is in this neighborhood, then $h_{t}$ and $h_{u}$ are homotopic, considered as maps into $E_{n}-N$. But the inclusion maps of $E_{n}-N$ into $E_{n}-x$ and $E_{n}-y$ induce homology isomorphisms. Thus $h_{t}$ links $x$ if and only if $h_{u}$ links $y$, and the proof is complete.

Received by the editors March 22, 1957.

1 The second author was supported in part by a grant from the National Science Foundation. 
A subset $A$ of a space $X$ will be said to separate two points $a$ and $b$ of $X$ provided every compact connected subset $F$ of $X$ containing $a$ and $b$ meets $A$.

LEMma 2. Let the points $a$ and $b$ of a compact space $X$ be scparated by a closed subset $A$ of $X$. If the homology group $H_{1}(X)$ is zero, then there is a compact connected subset $K$ of $A$ which separates $a$ and $b$.

Proof. An application of Zorn's lemma shows that there is a minimal compact subset $K$ of $A$ which separates $a$ and $b$. But $K$ is connected, for if not $K$ can be expressed as the union of two disjoint nonempty closed subsets of $X$ neither of which separates $a$ and $b$. However this would contradict Theorem VII 9.2 of [1].

Lemma 3. If $T: X \rightarrow Y$ is a continuous point-to-set function from a compact connected space $X$ onto a compact space $Y$ and if $L$ is a component of $Y$, then $T^{-1}(L)=X$.

Proof. The set $L$ is the intersection of its open-and-closed neighborhoods $N_{\alpha}$, and since $T$ is continuous and $X$ connected, $T^{-1}\left(N_{\alpha}\right)=X$ for each $\alpha$. Thus the closed sets $T(x) \cap N_{\alpha}$ are all nonempty, and for a particular $x \in X$ the collection $\left\{T(x) \cap N_{\alpha}\right\}$ has the finite intersection property, so that $T(x)$ meets $L$.

PROOF OF THE THEOREM. Identify the points $(x, t)$ of $C \times E_{1}$ for which $t \geqq 2$ (call this point $\alpha$ ) and the points for which $t \leqq-1$ (call this point $\beta$ ). The resulting space $\widetilde{C}$ is the two-point suspension of $C$. Using the Mayer-Vietoris sequence [2] one finds that since $C$ is connected, $H_{1}(\widetilde{C})=0$. By Lemma $1, B^{*}$ separates $\alpha$ and $\beta$ in $\tilde{C}$, and thus, by Lemma 2 , there is a compact connected subset $K$ of $B^{*}$ which also separates $\alpha$ and $\beta$. The function $T \times 1: C \times E_{1} \rightarrow C \times E_{1}$ is continuous and determines in a natural way a continuous function $\tilde{T}: \widetilde{C} \rightarrow \widetilde{C}$. Let $L$ be a component of the compact space $T(K)$. Suppose $L$ and $K$ are disjoint. By Lemma $3 \widetilde{T}^{-1}(L)=K$; hence in particular $K$ and $L$ have the same projections on $E_{1}$. Thus there are arcs in $\tilde{C}-K$ from $\alpha$ and $\beta$ to $L$. But this contradicts the fact that $K$ separates $\alpha$ and $\beta$, hence $L$ meets $K$. Thus there is a $t, 0<t \leqq 1$, such that $T\left(h_{t}(B)\right)$ meets $h_{t}(B)$.

Note that the theorem remains true if $T$ is an upper semi-continuous function such that $T(x)$ is connected for each $x$, for then $\tilde{T}(K)$ is itself connected and can replace $L$ in the preceding proof.

2. Applications. 1. Let $B$ be an $n$-sphere in $E_{n+1}$ and $C$ a (concentric) $n$-sphere in the interior of $B$. Let $d \leqq \operatorname{diam} C$ and let $h_{t}$ be as in the Theorem. Then some $B_{t}=h_{t}(B)$ will intersect $C$ in points $x, y$ with $d(x, y)=d$. 
Proof. Let $C_{x}$ be the set of points on $C$ whose distance from $x$ is $d$. Then the mapping $T(x)=C_{x}$ is continuous.

REMARK. We can define the function $t(x)$ on $C$ as the "time" $t$ at which $x \in B_{t}$. This function is not necessarily single valued and therefore usually upper semi-continuous only but it is a function with connected graph. Thus the theorem of Kakutani-Yamabe-Yujobo [3] can be generalized for this function to yield that for some $t$ the set $B_{t} \cap C$ contains the endpoints of $n+1$ mutually orthogonal radii of $C$.

2. W. Gustin has raised the following question. Given a convex surface $C$ in $E_{3}$ what is the minimal length of a closed curve that can be "slipped over" $C$ ? He remarked that the minimal perimeter of all orthogonal projections of $C$ is obviously long enough to be slipped over $C$ even without bending. I. Schoenberg has shown that this length is not minimal even for tetrahedra and conjectured that the minimal length is that of the minimal closed geodesic on $C$. There are good heuristic arguments in favor of this conjecture, but it has not been proved completely so far. ${ }^{2}$

With the help of our theorem we can obtain some information on the problem even without the assumption that $C$ is convex.

The process of "slipping over" can be replaced by a varying simple closed curve $K_{t}(0 \leqq t \leqq 1)$ on $C$ whose closed interior expands from a point for $K_{0}$ to the whole of $C$ for $K_{1}$. This process can in turn be replaced by the shrinking of a surface $B$ which contains $C$ in its interior to a point in the interior of $C$, if we replace the interior of $K_{t}$ on $C$ by its image under a slight radial contraction towards a point $P$ in the interior of $C$, and the exterior of $K_{t}$ on $C$ by its image under a slight radial dilation from $P$. We then connect the two pieces by the necessary part of the cone through $K_{t}$ with vertex $P$.

Thus we can rephrase the problem. Let $B$ be a closed surface containing the convex surface $C$. Let $B$ be shrunk to a point so that $B_{t} \cap C$ is a rectifiable simple curve $K_{t}$, and let $l$ be the maximum of the length of $K_{t}$. Then what is the minimum of $l$ for all possible contractions $B_{t}$ ?

From our theorem we know that for every continuous transformation $T$ of $C$ into itself, $K_{t}$ will have to pass through a point $x$ and intersect $T(x)$ for some $t$. Thus the mapping into diametrically opposite points proves Gustin's curves to be minimal for spheres and some other centrally symmetric surfaces (such as right circular cylinders and ellipsoids).

${ }^{2}$ Added in proof. Schoenberg's conjecture has been proved by H. Busemann and will appear in his book Convex surfaces, Interscience Publishers, New York. 
We may obviously restrict our attention to arbitrarily smooth surfaces $C$. For such surfaces we can define the continuous mapping $T$ which maps every point $x$ into the set of points whose geodesic distance from $x$ is no less than that of the nearest conjugate point of $x$. Thus we obtain that $l$ is no less than twice the distance between the nearest conjugate points on $C$.

This investigation arose from a more special theorem of C. B. Tompkins. We wish to thank Arnold Shapiro for valuable suggestions.

\section{REFERENCES}

1. R. L. Wilder, Topology of manifolds, New York, 1949.

2. S. Eilenberg and N. Steenrod, Foundations of algebraic topology, Princeton, 1952.

3. H. Yamabe and Z. Yujobo, On the continuous function defined on a sphere, Osaka Mathematical Journal vol. 2 (1950) p. 19.

The University of California at Los Angeles and

The Institute for Advanced Study 